\title{
ANÁLISE DA ATOMIZAÇÃO GIRATÓRIA EM REATORES DE SPRAY FLAMENJANTE POR TÉCNICAS DE CFD
}

\author{
A. C. KLEMZ ${ }^{1}$, H. RAMLOW ${ }^{1}$, W. G. de M. GONÇALVES ${ }^{1}$ D. NORILER, H. F. MEIER ${ }^{1}$, \\ U. FRITSCHING ${ }^{2}$ \\ ${ }^{1}$ Universidade Regional de Blumenau, Departamento de Engenharia Química \\ ${ }^{2}$ Universidade de Bremen, Departamento de Engenharia de Processos \\ E-mail: anacarolinek@gmail.com
}

\begin{abstract}
RESUMO - A aplicação de nanomateriais tem recebido especial atenção devido a suas propriedades físicas e químicas e suas diversas aplicações. Em comparação com os métodos convencionais de produção de nanopartículas, o processo Flame Spray Pyrolysis (FSP) tem a vantagem de que os materiais de partida são dissolvidos diretamente em um combustível e, portanto, podem ser facilmente fornecidos à zona de reação. Desta forma, a compreensão abrangente do processo FSP e do efeito de suas variáveis sobre as características das nanopartículas formadas é de fundamental importância para o desenvolvimento de técnicas de produção em larga escala. O objetivo deste trabalho é avaliar a atomização giratória em reatores de spray flamejante por aplicação das técnicas de fluidodinâmica computacional (CFD). Os principais resultados mostram uma validação qualitativa da forma do spray a frio, a morfologia da chama e campos de velocidade e temperatura para duas distintas configurações. Observou-se que o atomizador $30^{\circ} / 15^{\circ}$ apresentou uma largura de chama $4 \%$ maior e uma altura $2,8 \%$ maior em relação ao atomizador $30^{\circ} / 5^{\circ}$, além de um alcance da zona de evaporação de 2,5 vezes maior.
\end{abstract}

Palavras-chave: Produção de Nanopartículas, Fluidodinâmica Computacional, Modelagem Matemática, Spray Flamejante (FSP), Atomização Giratória.

\section{INTRODUÇÃO}

A importância das nanopartículas aumentou nos últimos anos devido as suas propriedades físicas e químicas e diversas aplicações tais como, catálise (Fischer-Tropsch), células fotovoltaicas $\left(\mathrm{TiO}_{2}\right)$, sensores de gás, indústria de pigmentos $\left(\mathrm{TiO}_{2}\right)$ entre outras. Devido a todas estas aplicações, cresce a necessidade de um processo de fabricação mais eficiente, em maior escala (Ramlow, 2013). Neste sentido, o processo Flame Spray Pyrolysis (FSP) apresenta-se como uma técnica altamente promissora e versátil para a síntese rápida e em boa escala de nanomateriais com aplicações em engenharia. O Processo FSP foi introduzido em 2002 por Mädler et al. e baseia-se na utilização exclusiva de precursores líquidos altamente exotérmicos, em particular os baseados em solventes orgânicos, que dão origem às chamas autossustentáveis. Um precursor é um componente químico no qual o elemento desejado é incorporado e liberado durante a combustão, sob a forma de partículas. A mistura do "precursor e combustível" é pulverizada através de um bico para uma zona de reação e lá é atomizado a partir de um gás de 
dispersão. O líquido atomizado torna-se inflamado e forma uma chama de pulverização autossustentável (Mädler et al., 2002). Devido à natureza altamente exotérmica dos líquidos precursores no processo de FSP, as temperaturas de chama chegam de 2600 a $2800 \mathrm{~K}$. Ao mesmo tempo, elevadas velocidades de gás de arraste no processo de FSP, induzem o arraste radial do gás circundante juntamente com a perda de calor por radiação, o que dá origem a tempos de permanência extremamente curtos (milissegundos) com altos gradientes de temperatura ao longo do eixo da chama. A interação entre a elevada temperatura e um elevado gradiente de temperatura é uma das características mais importantes do processo FSP: a alta temperatura local promove a formação de materiais homogêneos e altamente cristalinos e também promove o crescimento das partículas por coalescência e sinterização, enquanto que o elevado gradiente de temperatura e o curto tempo de residência preservam a funcionalidade das partículas de escala manométrica (Teoh et al., 2010).

Dado que o tempo de residência das nanopartículas dentro da chama é um parâmetro determinante para a sua formação, tem-se que um maior ângulo de spray e uma chama maior e mais ampla, proporcionadas pelas taxas de produção mais elevadas, tem influência direta nas características das nanopartículas formadas (Meier et at., 2014). Neste sentido, a rotação (swirl), além de ser importante para promover a estabilidade da chama, aumentar a eficiência de combustão e controle de emissões (Gupta et al., 1984), ainda permite manter os tempos de residência das nanopartículas no interior da chama por alteração das características morfológicas da chama. Assim, a utilização de atomizadores giratórios surge como uma solução possível, sendo utilizado em diversos processos, tais como a injeção de combustível em motores de combustão motores de combustão interna, caldeiras, turbinas a gás, fornos e caldeiras (HILL et al., 1994).

O presente trabalho tem por objetivo avaliar a atomização giratória em reatores de spray flamejantes por aplicação das técnicas de fluidodinâmica computacional (Computational Fluid Dynamics - CFD). Duas combinações de atomizadores foram analisadas, sendo este formado por um bico injetor líquido e alimentação de gás de dispersão por oito pontos de injeção cujo componente de giro é promovido pela torção dos injetores $(\beta)$ de $5^{\circ}$ e $15^{\circ}$.

\section{MATERIAL E MÉTODOS}

\subsection{Modelo Matemático}

Para a modelagem do processo algumas condições foram implementadas. O modelo considera fase gás contínua numa abordagem Euleriana (equações de continuidade, momento, energia e espécies químicas) e a fase dispersa de gotículas numa abordagem Lagrangeana (balanços de energia, força e massa) através de um acoplamento de duas vias, sob condições de estado estacionário e escoamento incompressível. A formação das nanopartículas depois da vaporização e da combustão do precursor líquido na chama do spray e sua dinâmica não têm qualquer influência sobre o fluxo multifásico e não são considerados. Apenas a força de arraste é considerada com coeficiente de arraste calculado a partir da equação apresentada por Morsi e Alexander (1972). A transferência de calor e massa entre as fases, incluindo vaporização, são consideradas. Os números de Nusselt e Sherwood são avaliadas pela correlação de Ranz e Marshall (1952). A radiação é considerada pelo modelo P-1. As condições de turbulência são 
representados pelas equações médias de Navier-Stokes assumindo a hipótese de viscosidade turbulenta, calculado pelo modelo de turbulência (SST) k- $\omega$. A fase de gotículas dispersas é representada pelo modelo de distribuição de tamanho de Rosin-Rammler-Sperling-Bennet (RRSB). Um modelo estocástico chamado Discret Random Walk (DRW) foi utilizado para prever a dispersão de gotículas turbulentas. A combustão é assumida como limitada pela mistura, e, assim, os efeitos da turbulência sobre a taxa de reação devem ser considerados. Magnussen e Hjertager (1977) assumem que a taxa de combustão é determinada pela taxa de transferência de massa entre as escalas de vórtices reagentes, e partindo desse pressuposto, desenvolveram modelo de "Eddy Dissipation Concept" (EDC), sendo este aplicado no presente trabalho. Detalhes da modelagem matemática podem ser encontrados em Noriler et al., 2014.

\subsection{Condições Geométricas e Operacionais}

Duas combinações de atomizadores foram analisadas, sendo estes formados por um bico injetor de líquido e alimentação de gás de dispersão por oito pontos de injeção dispostos em um círculo de raio $\mathrm{D}$ em volta do injetor de líquido conforme a Figura 1 . O componente de giro é promovido pela torção dos injetores de gás de dispersão em ângulos $(\beta)$ pré-definidos de $5^{\circ}$ e $15^{\circ}$. Além disso, o componente radial de velocidade é promovido pela inclinação $(\alpha)$ de $30^{\circ}$ dos dutos de dispersão de gás em relação ao eixo axial, conforme apresentado na Figura 1 (direita).

Etanol como combustível, $\mathrm{O}_{2}$ como gás de dispersão, e uma mistura $\mathrm{CH}_{4} / \mathrm{O}_{2}$ para as chamas suporte foram utilizados como fluidos de processo. As gotículas do spray são injetadas no domínio computacional com posição, velocidade e distribuição de tamanho obtidas experimentalmente por Klemz (2013) que verificou a geometria do cone de líquido por imagens de câmera de alta velocidade e distribuição de tamanho por espectroscopia de difração a laser. A Tabela 1 apresenta as condições de contorno utilizadas nas simulações.

Tabela 1 - Condições de contorno

\begin{tabular}{c|c|c}
\hline Propriedade & Atomizador $5^{\circ} / 30^{\circ}$ & Atomizador $15^{\circ} / 30^{\circ}$ \\
\hline Altura do cone & $7,43 \mathrm{~mm}$ & $9,78 \mathrm{~mm}$ \\
\hline Ângulo do cone & $26,23^{\circ}$ & $13,43^{\circ}$ \\
\hline Raio da base do cone & $1,686 \mathrm{~mm}$ & $1,118 \mathrm{~mm}$ \\
\hline Diâmetro mínimo & $2,12 \mu \mathrm{m}$ & $2,12 \mu \mathrm{m}$ \\
\hline Diâmetro máximo & $393,45 \mu \mathrm{m}$ & $1.125,570 \mu \mathrm{m}$ \\
\hline Parâmetro de escala RRSB $(\bar{d})$ & $19,745 \mu \mathrm{m}$ & $36,08 \mu \mathrm{m}$ \\
\hline Parâmetro de forma RRSB $(n)$ & 1,96 & 1,16 \\
\hline Velocidade & $27 \mathrm{~m} / \mathrm{s}$ & $27 \mathrm{~m} / \mathrm{s}$ \\
\hline Taxa total de etanol & $250 \mathrm{ml} / \mathrm{min}$ & $250 \mathrm{ml} / \mathrm{min}$ \\
\hline Taxa total de $\mathrm{O}_{2}$ & $250 \mathrm{Nl} / \mathrm{min}$ & $250 \mathrm{Nl} / \mathrm{min}$ \\
\hline Chama piloto $\mathrm{CH}_{4} / \mathrm{O}_{2}(86 / 142)$ & $228 \mathrm{nl} / \mathrm{min}$ & $228 \mathrm{nl} / \mathrm{min}$
\end{tabular}

A distribuição de partículas é representada por uma distribuição de RRSB, definida pela Equação 1,

$$
Y_{d}=\exp \left[-\left(\frac{d}{\bar{d}}\right)^{n}\right]
$$


onde $\bar{d}$ é o parâmetro de escala e $n$ é o parâmetro de forma da distribuição RRSB.

\section{SIMULAÇÃO NUMÉRICA}

As simulações numéricas foram realizadas inicialmente considerando spray a frio, ou seja, sem a evaporação e combustão, a fim de validar o modelo por comparação com a forma do spray obtida experimentalmente por Klemz (2013) e, em seguida, realizar simulações numéricas com os efeitos de transferência de calor, massa e reação química para investigar diferentes condições de atomização sobre a morfologia e o perfil de temperatura da chama.

\subsection{Metodologia Numérica}

A partir das geometrias foram criadas as malhas tridimensionais com elementos hexaédricos utilizando o software "ICEM 14.0", conforme apresentada na Figura 1 (esquerda). A malha do atomizador $5^{\circ} / 30^{\circ}$ possui 1.514 .976 nós, um ângulo mínimo de $37,98^{\circ}$ e um determinante mínimo de 0,81 . A malha do atomizador $15^{\circ} / 30^{\circ}$ possui 1.533 .978 nós, um ângulo mínimo de $37,71^{\circ}$ e um determinante mínimo de 0,8 .
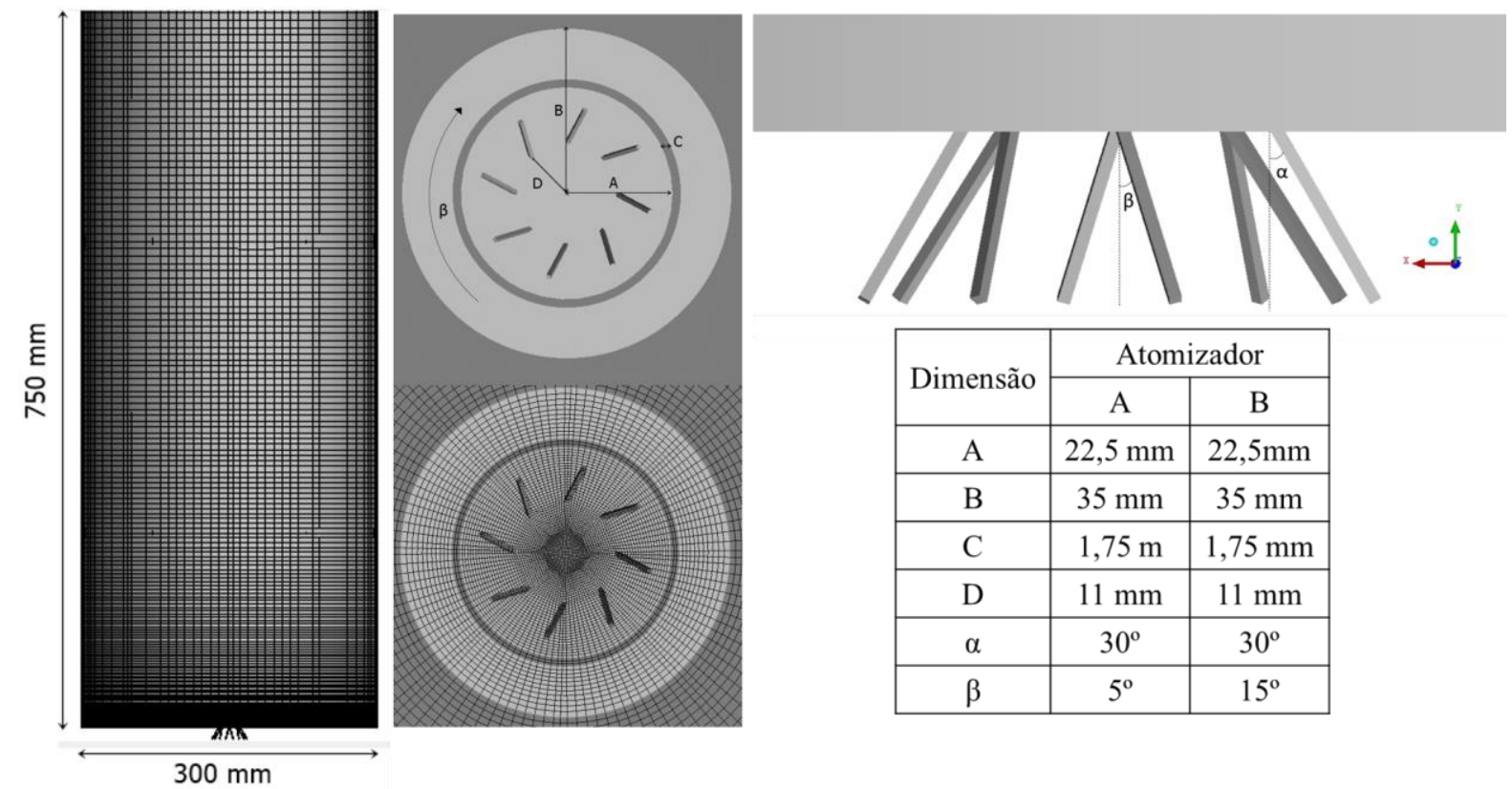

Figura 1 - Geometria e malha numérica do reator de spray flamejante.

As simulações numéricas foram executadas no software FLUENT 14.0 através da implementação da geometria, da malha numérica e do modelo Euleriano-Lagrangeano. Primeiramente as simulações foram realizadas sem reação e com temperatura da entrada de metano e de oxigênio de $300 \mathrm{~K}$ para 1000 interações. Após isso, foi ativada a reação com temperatura de entrada de metano e de oxigênio de $3000 \mathrm{~K}$ para 500 interações. Por fim, diminui-se a temperatura de entrada de metano e de oxigênio para $300 \mathrm{~K}$ para 3000 interações, até que a convergência fosse atingida. 


\section{RESULTADOS E DISCUSSÕES}

A Figura 2 apresenta os resultados das simulações a frio dos campos de velocidade e trajetória das gotículas comparadas às imagens experimentais obtidas por Klemz (2013). Os principais resultados mostram que o aumento do ângulo de giro $\beta$ modifica o padrão de fluxo do gás de arraste. Com maior velocidade tangencial na fase gasosa, o spray fica maior e o fluxo total de massa de ar na direção axial aumenta, conforme por ser observado na Figura 2 à esquerda, que mostra os campos de velocidade vetorial da fase gasosa em um plano central sobrepostas às imagens experimentais. Dados experimentais (Klemz, 2013) mostraram a deposição das gotículas na base do reator nos experimentos com atomizadores $\operatorname{com} \beta=5^{\circ}$. Esse comportamento também foi observado na investigação numérica para as gotículas menores, conforme apresentado na Figura 2 ao centro. No entanto, isto ocorreu em apenas cerca de 2,5 $\%$ das gotículas, o que em termos de fluxo de massa do spray é relativamente pouco significativo $\left(3,4 \times 10^{-5} \%\right)$. Já para o ângulo $\beta=15^{\circ}$ este comportamento não foi observado. Uma boa validação qualitativa da forma do spray foi alcançada sobrepondo as trajetórias simuladas com imagens de câmera de alta velocidade para ambos os casos, conforme pode ser observado na Figura 2 à direita.

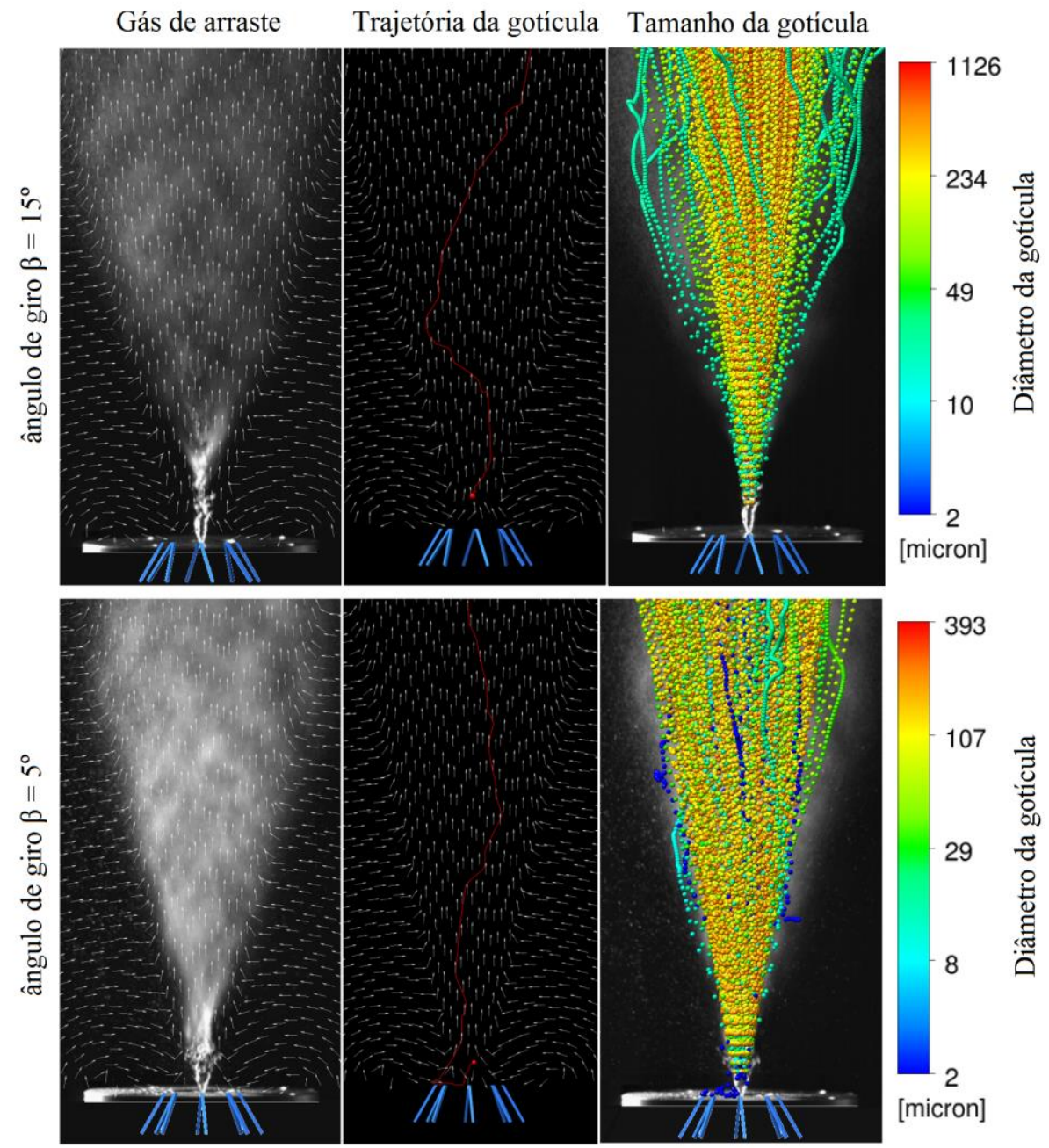

Figura 2 - Campos de velocidade e trajetória de gotículas sobrepostas à imagens experimentais (Klemz, 2013). 

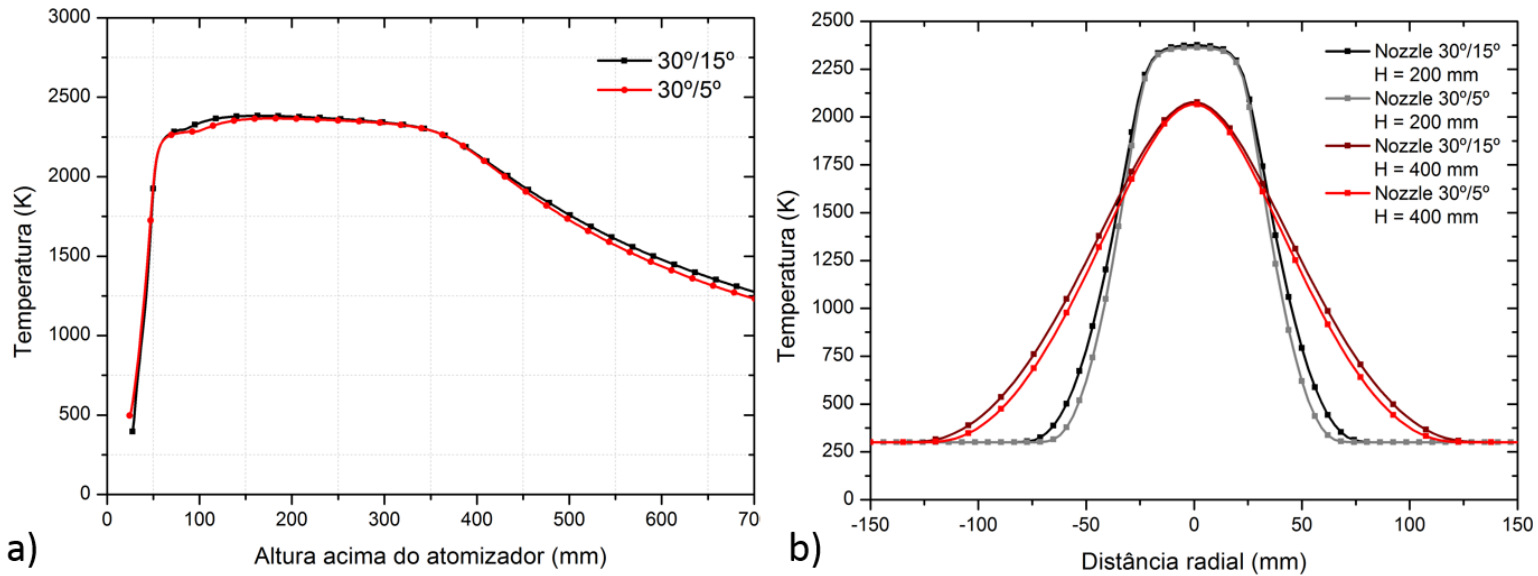

Figura 3 - Perfis de temperatura axial e radial para o spray reativo.

As Figuras 3 e 4 apresentam os resultados obtidos pela simulação do spray reativo. A Figura 3 apresenta os os perfis de temperatura em uma linha central desde a base do atomizador até o topo do reator, Figura $3 \mathrm{a}$, e os perfis de temperatura em uma linha perpendicular a base do reator a $200 \mathrm{~mm}$ e $400 \mathrm{~mm}$ de altura, Figura $3 \mathrm{~b}$, para os atomizadores $30^{\circ} / 5^{\circ}$ e $30 \% 15^{\circ}$. Podese observar que os perfis de temperatura são similares com pequenas diferenças significativas no perfil axial apartir de $450 \mathrm{~mm}$. Já para os perfis radiais de temperatura, nota-se que para ambas alturas o atomizador $30^{\circ} / 15^{\circ}$ apresenta maior espalhamento devido a maior intensidade do campo centrífugo (swirl). O resultado deste padrão pode ser melhor observado na Figura 4, que apresenta as isosuperfícies de temperatura a $1500 \mathrm{~K}$ e concentração de gotículas $1 \times 10^{-3}$ $\mathrm{kg} / \mathrm{m}^{3}$. A isosuperfície de temperatura representa a morfologia da chama visível (Müller et al ., 2004). Nota-se que a chama promovida pelo atomizador $30^{\circ} / 15^{\circ}$ é $4 \%$ mais larga, devido a maior intensidade do campo centrífugo, e $2,8 \%$ mais alta.

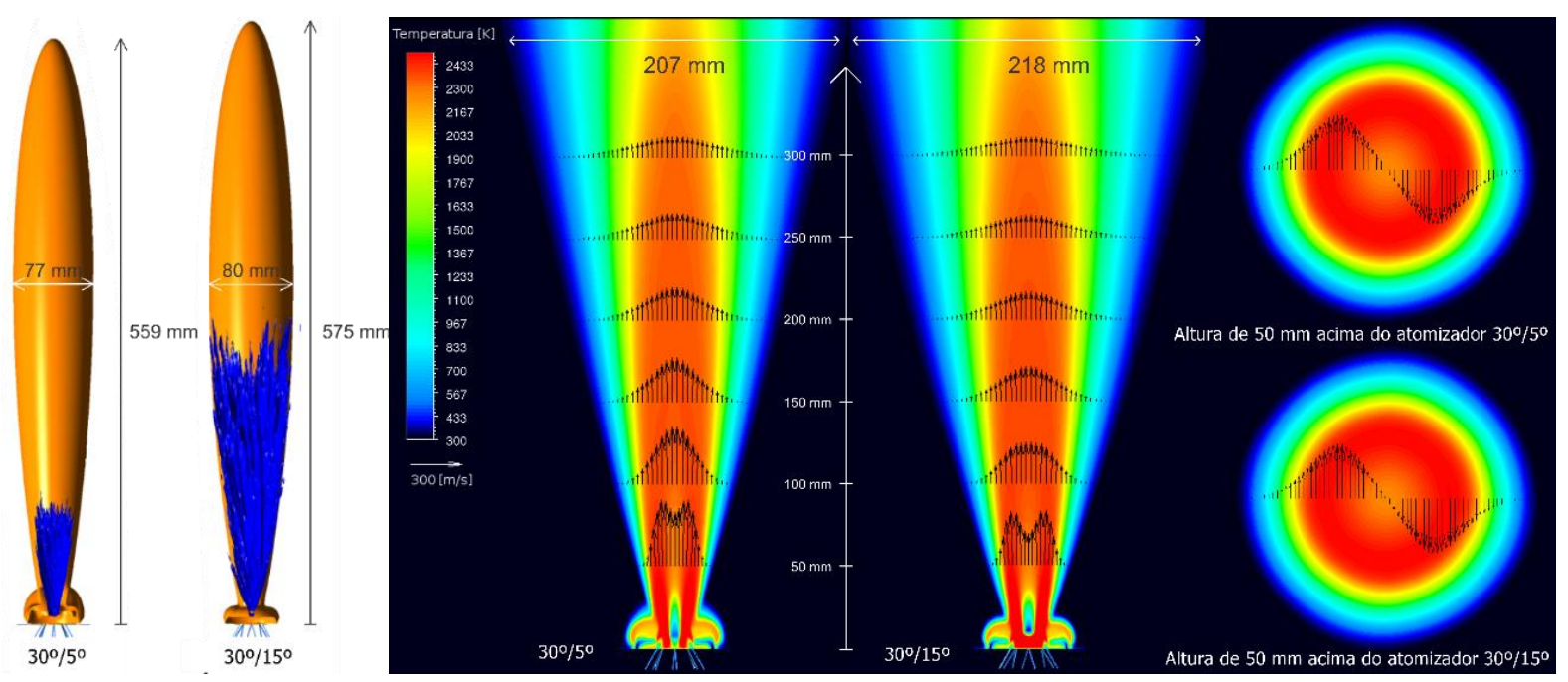

Figura 4 - Morfologia da chama e do spray e campos de velocidade e temperatura para o spray reativo. 
A maior altura pode ser explicada pelo alcance do spray no interior da chama. Notase, por observação das isosuperfícies de concentração na Figura 4, que a evaporação principal se extende até a metade da chama para o atomizador $30^{\circ} / 15^{\circ}$, ao passo que para o atomizador $30^{\circ} / 5^{\circ}$ a evaporação principal ocorre até $1 / 5$ da chama. Isto ocorre, provavelmente, porque a distribuição de tamanho das gotículas produzidas pelo atomizador $30^{\circ} / 5^{\circ}$ apresenta menores diâmetros. Observa-se ainda na Figura 4, que o atomizador $30^{\circ} / 15^{\circ}$ apresenta maior espalhamento do campo de temperatura, sendo este motivado pela maior amplitude dos campos de velocidade tangencial, observados a $50 \mathrm{~mm}$ da base do atomizador.

\section{CONSIDERAÇÕES FINAIS}

O processo FSP apresenta-se como um método versátil para a produção de nanopartículas de óxidos metálicos. Com o objetivo de compreender o efeito da atomização giratória sobre reatores de spray flamejante, simulações numéricas foram realizadas para determinar o comportamento e a morfologia do spray a frio, a morfologia da chama e os campos de velocidade e temperatura do spray reativo.

Os principais resultados mostram uma boa concordância da forma do spray a frio quando comparadas imagens obtidas em bancadas experimentais com as trajetórias preditas pelo modelo. $\mathrm{O}$ atomizador $30^{\circ} / 5^{\circ}$ apresentou deposição de gotículas na base do reator, mesmo que em pequenas proporções.

Para ambos atomizadores os perfis de temperatura axial são similares com pequenas divergências próximo a base do reator e a partir de $450 \mathrm{~mm}$ de altura. Já para os perfis radiais de temperatura o atomizador $30^{\circ} / 15^{\circ}$ apresentou maior espalhamento do campo de temperatura, promovido pela maior intensidade do campo centrífugo. $\mathrm{O}$ atomizador $30^{\circ} / 15^{\circ}$ apresentou uma chama $4 \%$ mais larga e 2,8\% mais alta, além de um maior alcance da zona de evaporação, correspondente a metade da altura da chama $(\sim 2,5$ vezes maior que o obtido pelo atomizador $\left.30^{\circ} / 5^{\circ}\right)$.

\section{REFERÊNCIAS}

DUBEY, R., BHADRAIAH, K., AND RAGHAVAN, V., On the estimation and validation of global single-step kinetics parameters of etanol air oxidation using diffusion flame extinction data, Combustion Science and Technology, vol. 183, no. 1, pp. 43-50, 2011.

GUPTA AK, LILLEY DG, SYRED N. Swirl flows. Tunbridge Wells (England): Abacus Press; 1984.

HILL PG, ZHANG D. A review of swirling flow and combustion in spark-ignition engines. Prog Energy Combust Sci 1994; 20: 373-429.

JONES, W. P.,LINDSTEDT, R. P., Global reaction schemes for hydrocarbon combustion, Combustion and Flame, vol. 73, no. 3, pp. Atomization and Spray Parameters on the FSP. 
KLEMZ, A. C. Investigação experimental e comparação de dois sistemas de atomização para a produção de nanopartículas. Blumenau: Universidade Regional de Blumenau; Agosto, 2013.

MÄDLER, L., KAMMLER, H. K., MUELLER, R., PRATSINIS, S. E., 2002. Controlled synthesis of nanostructured particles by flame spray pyrolysis. Journal of Aerosol Science 33 (2), 369-389.

MAGNUSSEN, B. F., HJERTAGER, B. H., On mathematical models of turbulent combustion with special emphasis on soot formation and combustion, 16th Symposium (International) on Combustion, Vol. 16, The Combustion Institute, pp. 719-729, 1977.

MEIER, H. F., MORI, M., MÄDLER, M., FRITSCHING, U., Manufacturing process of nanostructured deposition coatings. Blumenau, 2014.

MORSI, S. A., ALEXANDER, A. J., An investigation of particle trajectories in two-phase flow systems, Journal of Fluid Mechanics, 18 vol. 55, no. 2, pp. 193-208, 1972.

MUELlER, R., JOSSEN, R., KAMMLER, H. K., PRATSINIS, S. E., AKHTAR, M. K. Growth of zirconia particle made by flame spray pyrolysis. AICHE Journal, 50, pp. 30853094, 2004.

NORILER, D., ROSEBROCK, C., POKHREL, S., MÄDLER, L., MEIER, H. F., FRITSCHING, U., 2011. Spray structural parameter influence on the flame spray pyrolysis (FSP) process for nanoparticle production. In: 24th European Conference on Liquid Atomization and Spray Systems. ILASS Europe 2011.

NORILER, D., ROSEBROCK, C., Pokhrel, S., MÄDLER, L., MEIER, H. F., FRITSCHING, U.Influence of atomization and spray parameters on the Flame Spray process for nanoparticle production. Atomization and Sprays, vol. 24, no. 6, pp. 495-524, 2014.

RAMLOW, H. Experimentelle charakterisierung von sprays mit variation der zerstäubergeometrie, massenströmen und geometrischen randbedingungen. Bremen, 2013.

RANZ, W. E., MARSHALL, W. R., Evaporation from drops, part i and part ii, Chemical Engineering Progress, vol. 48, no. 4, pp. 173-180, 1952.

TEOH, W.Y., AMAL, R. \& MAEDLER, L., 2010, Flame spray pyrolysis: An enabling technology for nanoparticles design and fabrication, Nanoscale, 2, 8, 1324-1347. 